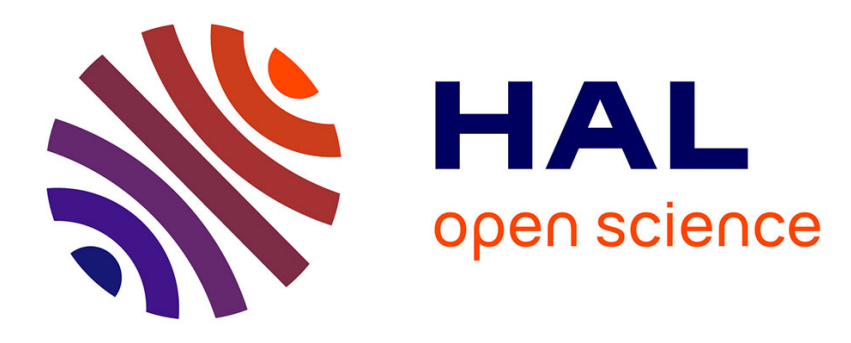

\title{
Hybrid Spectrum Sharing through Adaptive Spectrum Handoff and Selection
}

\author{
Adisorn Lertsinsrubtavee, Naceur Malouch
}

\section{To cite this version:}

Adisorn Lertsinsrubtavee, Naceur Malouch. Hybrid Spectrum Sharing through Adaptive Spectrum Handoff and Selection. IEEE Transactions on Mobile Computing, 2016, 15 (11), pp.2781 - 2793. 10.1109/TMC.2016.2517619 . hal-01400064

\section{HAL Id: hal-01400064 https://hal.sorbonne-universite.fr/hal-01400064}

Submitted on 21 Nov 2016

HAL is a multi-disciplinary open access archive for the deposit and dissemination of scientific research documents, whether they are published or not. The documents may come from teaching and research institutions in France or abroad, or from public or private research centers.
L'archive ouverte pluridisciplinaire HAL, est destinée au dépôt et à la diffusion de documents scientifiques de niveau recherche, publiés ou non, émanant des établissements d'enseignement et de recherche français ou étrangers, des laboratoires publics ou privés. 


\title{
Hybrid Spectrum Sharing Through Adaptive Spectrum Handoff and Selection
}

\author{
Adisorn Lertsinsrubtavee and Naceur Malouch
}

\begin{abstract}
Spectrum sharing is a key function to provide fairness allocation as well as service satisfaction across multiple users in cognitive radio networks. Even though spectrum sharing can benefit from spectrum handoff to enhance rate performance by switching from unavailable channels to available ones, the negative impact on handoff delay can cause significant service degradation. In this work, we present a hybrid spectrum sharing strategy that includes novel static and dynamic spectrum sharing algorithms based essentially on a rate compensation approach and adapted best fit algorithms. The static scheme is applicable for some specific network configurations where spectrum handoff is not necessary. Conversely, the dynamic scheme can benefit from spectrum handoff to increase the achieved rate and also compensate for the lost rate from the unavailable periods. These two sharing schemes are operated adaptively according to the current network environment. We compare our hybrid strategy with a fully dynamic one and an optimization framework. The proposed hybrid spectrum sharing demonstrates its effectiveness in terms of improving the overall service satisfaction and reducing the number of handoffs while the achieved rate is fulfilling compared to the optimal.
\end{abstract}

Index Terms-Cognitive Radio Networks, Spectrum Sharing, Spectrum Handoff, Rate Compensation, Static Spectrum Sharing, Dynamic Spectrum Sharing, Rate Requirement, Best Fit Algorithm.

\section{INTRODUCTION}

$\mathrm{T}$ HE significant underutilization of the licensed spectrum is posing new challenges related to the design of new network paradigms for wireless communication. As a consequence, Cognitive Radio Networks (CRNs) are proposed in order to improve the spectrum utilization by allowing secondary users (SUs) to temporarily occupy the spectrum bands that are unused by primary users (PUs). One of the challenges in CRNs is related to high fluctuations in available spectrum so that the service requirement of SUs is hard to achieve, especially when multiple SUs must compete to share limited and dynamic spectrum bands. Therefore, efficient spectrum sharing is necessary to provide fairness allocation as well as service satisfaction across multiple users while maximizing the utilization of the total available bandwidth.

These goals are achieved concretely through spectrum handoff and spectrum selection functions while applying a given sharing strategy. The first possible strategy refers to static spectrum sharing where no handoff is performed during the transmission of an SU. This means the spectrum allocation is done only once, for instance, before starting the transmission. The second strategy refers to dynamic spectrum sharing where the rate allocation of SUs is recomputed instantaneously regarding $\mathrm{PUs}^{\prime}$ activities, for instance, when a frequency channel becomes unavailable [1].

The static spectrum sharing strategy is usually applied with the centralized architecture where a CR base station has global information on the network and decides optimally

- A. Lertsinsrubtavee is with Computer Laboratory, University of Cambridge,UK, E-mail:al773@cam.ac.uk

- N. Malouch is with the LIP6-NPA team, UPMC Sorbonne Universités, France, E-mail: Naceur.Malouch@lip6.fr.

- Part of this work was supported by the grant ANR-10-VERS-005-03. on the resource allocation for all SUs. There are several criteria for assigning spectrum to SUs, and these vary according to the target objective of each problem. One of the common objectives for spectrum sharing in CRNs refers to interference avoidance among SUs and also across to PUs. Many works in the literature have formulated linear-integer optimization problems with an objective to maximize the global throughput of SUs while limiting the transmission power under the interference temperature threshold (i.e., [2]-[4]). However, due to the constraint of the interference temperature, maximum spectrum utilization is not always ensured while in most cases the QoS is also not taken into account. Driven by this issue, a joint optimization framework is proposed to deal with the QoS and power control simultaneously [5], [6]. Nevertheless, the fairness issue is not considered in these works, such that a few SUs can satisfy their QoS but others are left with no available spectrum. To solve this unfairness problem, the traditional target objectives for max-min fairness and proportional fairness are applied in many works (i.e., [7]-[10]). Specifically, static spectrum sharing can avoid the impacts of spectrum handoff delays by allowing SUs to backoff and wait if any of the PUs are using the same channel. If PUs occupy the channel for long periods, SUs would suffer for the long waiting delay leading to service degradations.

Dynamic spectrum sharing is addressed in [1], where the objective is to maximize the utilization of the spectrum. The user allocations are recomputed dynamically each time activities of PUs change the status of the spectrum. Similar to [11], [12], the channel reconfiguration algorithm based on the knapsack problem is introduced to optimize the number of SUs added in each time slot. The assigned time slot for SUs can be rearranged when the status of the spectrum is changed regarding the PU activities, and thus the global utilization can be maximized. However, these works do not 
contain any condition for controlling the number of spectrum handoffs. Therefore, it is expected that a large number of spectrum handoffs can be generated, which possibly impacts the rate performance, especially if a large spectrum handoff delay is considered. To control the impacts of spectrum handoff while maintaining the service requirement, a QoS driven approach has been addressed in few works [13], [14]. The handoff decision is not necessarily triggered by PU detection as in the classic proactive ( [15]-[17]) and reactive ([18]) approaches. Alternatively, the decision is made through an achieved performance of SU compared to a service requirement. In fact, an SU can backoff and wait for transmission, once any of the PUs or SUs start to contend in the same channel. Typically, SUs are encouraged to stay on the interrupted channel as long as the achieved performance satisfies the service requirement [19]. In this manner, SUs do not handoff frequently, and thus the disruption time which is caused by spectrum handoff delay can be alleviated. Furthermore, the network contention can be reduced, since SUs may have different service requirements. Thus, SUs may not contend with others to select the same channel at the same time.

Apart from those challenges which hinder CRNs to guarantee the service requirement of SUs, the number of wireless interfaces also introduced a new critical limitation in CRNs. Concretely, when SUs are equipped with a small number of wireless interfaces ${ }^{1}$, their service requirements may not be satisfied, even if the total available bandwidth is larger than the total demand of all SUs. The multi-channel communication is applied in several works to improve the quality of service (QoS) and the utilization through multiple wireless interfaces [1], [5], [20], [21]. Nevertheless, the limitation imposed by the number of wireless interfaces is as yet unexplored.

In our previous work [22], we explored the optimal rate allocation while considering multi-channel communications for SUs through the formulation of a global spectrum sharing (GSH) strategy where we assume global knowledge of channel states including their availability during the total communication period. Intuitively, the allocation could be optimized globally, where SUs may not perform spectrum handoff immediately when one of its current channels is not available. In fact, it can compensate its lost rate when the current channel becomes available again, or when it moves later to another available channel. Nevertheless, it is not always convenient to obtain complete longterm future PUs' activities in advance.

In this paper, we propose a heuristic for centralized spectrum sharing that exploits short-term future information on PUs' activities when they are provided. The main objective is to consider carefully the impact of spectrum handoff while trying to maximize user satisfaction in terms of achieved rates. Our main contributions are summarized in the following aspects:

- Instead of independently using the static or dynamic spectrum sharing, our heuristic is derived from both

1. In this work, we consider that a wireless interface is composed by a transceiver and one antenna. Thus, we use interchangeably the number of antennas and the number of wireless interfaces to indicate the number of possible parallel transmissions. approaches in a hybrid manner. It balances adequately the tradeoff between the benefits of spectrum handoff and the necessity of reducing their number. It maximizes the utilization of the network capacity and the achieved rates of SUs. This is done by modifying and adapting the renowned Best Fit algorithm which is commonly applied for the bin packing problem [23], [24], so that we consider originally multi-channel communications and spectrum handoff reduction.

- Apart from previous studies, our proposed strategy aims to maximize the service satisfactions while considering the fairness. We apply a two step allocation mechanism to the static spectrum sharing scheme to guarantee that the allocation can be satisfied for all SUs. For the dynamic spectrum sharing scheme, a priority channel selection is introduced to compensate lost rates by turns and achieve fairness in the long term.

- We evaluate the performance of our proposed hybrid spectrum sharing heuristic through simulations while considering the GSH optimization model [22] as a benchmark for the rate performance.

The rest of the paper is organized as follows. In section 2 , we describe the spectrum sharing model and highlight the impact of the number of wireless interfaces. A heuristic hybrid spectrum sharing strategy is explained in Section 3. In section 4, the performance of our strategies is evaluated. Finally, we give our concluding remarks in Section 5.

\section{Spectrum Sharing Model in Cognitive Ra- DIO NETWORKS}

We consider an infrastructure-based CRN with a total of $N$ secondary users (SU) and $M$ licensed channels available for opportunistic spectrum access. Each $\mathrm{SU}$ is equipped with $n$ wireless interfaces. A single SU can use multiple channels simultaneously through multiple wireless interfaces and each channel can be used by several SUs at the same time. The latter capability can be managed at the MAC layer of cognitive radio devices through various multiple spectrum access techniques such as random or time division access [25]. The estimated available bandwidth of channel $i$ is denoted by $B^{i}$. Each user $j$ has a different rate requirement $r_{j}$ (bps), which can be considered also as the user weight for sharing the available bandwidth. The sharing among SUs can be controlled through a CR base station that is responsible for protecting the primary network from possible interferences and degradations. In our proposed model, the CR base station can communicate and control all SUs through the common control channel similarly to the previous work [1]. When PUs appear in the licensed channel, the SUs' transmissions must stop or handoff to other available channels. If SUs stay on the interrupted channel, they continue transmission when PUs leave the channel. At a given time and according to some sharing criteria, each SU is allocated a bandwidth from each channel. $b_{j}^{i}$ denotes the allocated bandwidth for $S U_{j}$ over channel $i$, where $b_{j}^{i} \in \mathbb{R} ; 0 \leq b_{j}^{i} \leq B^{i}$. If $b_{j}^{i}=0$, then $S U_{j}$ is not tuned to channel $i$ and thus is not transmitting over this 
channel. The CR base station exerts control over all SUs to manage channel allocation. Fig. 1 shows an example that highlights the impact of the number of wireless interfaces on spectrum sharing strategy. It considers a scenario where two SUs compete to access three licensed channels in which available bandwidth is fixed to 4 . The rate requirements are 12 and 2 for $S U_{1}$ and $S U_{2}$ respectively. In scenario (a), both SUs are equipped with $n=2$ wireless interfaces. To maximize the total achieved rate of SUs, the allocation can give a rate of 4 from $\mathrm{Ch} 1$ and $\mathrm{Ch} 2$ to $S U_{1}$ and allocate a rate of 2 from Ch3 to $S_{2}$. In fact, there is still some available bandwidth over Ch3, but $S U_{1}$ cannot utilize this amount of bandwidth to satisfy its rate requirement. This is because the transmission of $S U_{1}$ is limited to 2 channels.

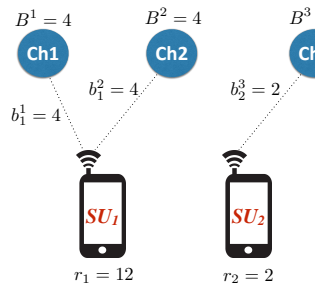

(a) Under-utilization
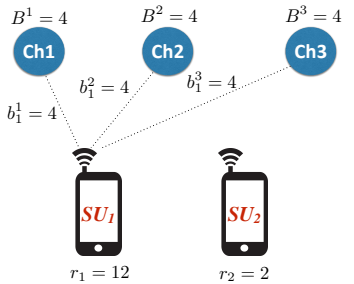

(b) Unfairness
Fig. 1: Spectrum sharing example with limited wireless interfaces

In scenario (b), we add another wireless interface to $S U_{1}$, thus $S U_{1}$ has $n=3$ and $S U_{2}$ has $n=2$ wireless interfaces. One of the solution to maximize the utilization is to allocate all three of the available channels to $S U_{1}$ and none to $S U_{2}$. Clearly, the number of wireless interfaces $(n)$ plays a vital role in the spectrum sharing strategy. In scenario (a), no optimization can maximize the utilisation since $S U_{1}$ could not satisfy its rate requirement, even though there is still some available bandwidth over Ch3. Nevertheless, in scenario (b) where the utilization of channels is maximized, the allocation is not fair to $S U_{2}$. As a matter of fact, the challenge is to design an effective spectrum sharing strategy which can achieve the service requirement for all SUs. Furthermore, the strategy should be able to share the available bandwidth fairly while considering different service requirements of SUs and capabilities in terms of wireless interfaces.

\section{Hybrid Spectrum Sharing Design}

In this section, we aim to propose a hybrid spectrum sharing heuristic that avoids the use of future information on PUs' activities and also reduces the number of spectrum handoffs. Concurrently, we consider the GSH strategy [22] as a reference model for the rate performance and develop a heuristic for spectrum sharing which contains two schemes of spectrum sharing including Static Spectrum Sharing (SSS) and Dynamic Spectrum Sharing (DSS). The former is inherited by the static approach to benefit from the case where a handoff is not necessary. The latter is derived from the dynamic strategy complemented by a rate compensation approach inspired from GSH. In addition, we have also applied the use of near future information on PUs' activities to this scheme. These two schemes are selected adaptively regarding the current network configurations. For clarity of representation, the important notations involved in the hybrid spectrum sharing are summarized in Table 1.

TABLE 1: Symbols used for the hybrid spectrum sharing

\begin{tabular}{|c|c|}
\hline Notations & Descriptions \\
\hline$M, N$ & Number of channels, Number of SUs \\
\hline$\Omega, S$ & Set of channels, Set of SUs \\
\hline$C H_{j}$ & Set of selected channels for $S U_{j}$ \\
\hline$E\left[T_{a v}^{i}\right]$ & Available periods of channel $i$ \\
\hline$E\left[T_{u n}^{i}\right]$ & Unavailable periods of channel $i$ \\
\hline$T_{\text {comp }}, T_{r}$ & Compensation period, Reservation period \\
\hline$b w^{i}$ & Link bandwidth of channel $i$ \\
\hline$\beta^{i}$ & Availability ratio of channel $i$ \\
\hline$\beta_{\text {Start }}^{i}$ & Initial availability ratio of channel $i$ \\
\hline$n_{j}$ & Number of antennas of $S U_{j}$ \\
\hline$r_{j}, a_{j}$ & Rate requirement of $S U_{j}$, Achieved rate of $S U_{j}$ \\
\hline$B^{i}$ & Available bandwidth of channel $i$ \\
\hline$B W_{l}$ & Total available bandwidth of a set of candidate channels \\
\hline$\alpha_{j}$ & Service satisfaction ratio of $S U_{j}$ \\
\hline$q_{j}(t)$ & Number of packets in transmission queue \\
\hline$r_{j}^{q}$ & $\begin{array}{l}\text { New Rate requirement of } S U_{j} \text { considering a number of } \\
\text { packets in the queue }\end{array}$ \\
\hline$r_{j}^{q^{2 n d}}$ & Rate requirement of $S U_{j}$ in the second step \\
\hline$b_{j}^{i}$ & Allocated rate for $S U_{j}$ over channel $i$ \\
\hline$c^{i}$ & Capacity of channel $i$ \\
\hline$C_{l}$ & Total capacity of a set of candidate channels \\
\hline$c_{j}^{i}, c_{r}^{i}$ & $\begin{array}{l}\text { Capacity of channel } i \text { after updating the } H O_{\text {delay }} \text { by } S U_{j} \text {, } \\
\text { Remaining capacity of channel } i\end{array}$ \\
\hline$l^{\max }$ & Maximum iteration in the channel selection process \\
\hline$A^{1 s t}$ & Effective maximum capacity of the channels \\
\hline$p_{j}, p_{j}^{\max }$ & Data to be sent, Maximum data to be sent \\
\hline & Quantity of data of $S U_{j}$ to be sent over channel $i$ \\
\hline $\operatorname{size}(q)$ & Size of $q^{t h}$ packet in the transmission queue \\
\hline$H O_{\text {delay }}, \Delta$ & Spectrum handoff delay, The maximum bound capacity \\
\hline$\chi$ & Occupied channel in the best fit iteration \\
\hline$\tau$ & Appropriate channel for replacing channel $\chi$ \\
\hline$k$ & Number of available periods in $T_{r}$ \\
\hline$h$ & Number of available periods during handoff \\
\hline$T H$ & Acceptable limit of availability ratio \\
\hline
\end{tabular}

\subsection{Static Spectrum Sharing}

The objective of Static Spectrum Sharing (SSS) is to satisfy the rate requirement of SUs for long-term communication without an attempt to perform spectrum handoff. Here, the $\mathrm{CR}$ base station determines the average total available bandwidth of selected channels to satisfy the constraint of the rate requirement $r_{j}$. To allocate the bandwidth properly, first we compute the average total available bandwidth based on the long-term availability ratio $\beta^{i}=\frac{E\left[T_{a v}^{i}\right]}{E\left[T_{a v}^{i}\right]+E\left[T_{u n}^{i}\right]}$ on each channel $i$, where $E\left[T_{a v}^{i}\right]$ and $E\left[T_{u n}^{i}\right]$ are the average of availability and unavailability periods of channel $i$ respectively. These values are obtained through long-term observations for each channel $i$. Then, the average long-term bandwidth can be computed as $B^{i}=\beta^{i} \cdot b w^{i}$, where $b w^{i}$ is the link bandwidth of channel $i$. It is worth mentioning that $B^{i}$ is an estimated available bandwidth from the past period based on PUs' activity. In our spectrum sharing algorithm, the base station will exploit this information to select and allocate the channel regarding the rate requirement of SUs. As the matter of fact, when a PU appears on the channel, SU will pause its on-going transmission immediately and wait for the new allocation either by staying on the same channel or by performing handoff to a new available channel. However, when we switch to this scheme, some SUs may not achieve sufficient rates from the prior allocation which can be reflected by the number of packets in their transmission 
queue $q_{j}(t)$. To take into account these packets in the queue, a new rate requirement $r_{j}^{q}$ is computed as follows:

$$
r_{j}^{q}=r_{j}+\frac{q_{j}(t)}{T_{\text {comp }}}
$$

where $T_{\text {comp }}$ is a compensation period during which packets from the queue shall be sent. We call it a compensation period because these packets correspond to a period where the allocated rate was lower than the requirement, and thus the lost rate must be compensated. Intuitively, the value of $T_{\text {comp }}$ should be large since the SSS is suitable for long-term communication without spectrum handoff.

To design an efficient heuristic algorithm for SSS, we are inspired by the Best Fit Decreasing (BFD) algorithm, which is frequently applied to solve the classic bin packing problem [23]. In this problem, a finite set of items must be packed into an infinite set of bins. Accordingly, in our spectrum sharing problem, a finite set of items is equivalent to a set of SUs denoted by $S$. As for a set of bins, it is equivalent to a set of channels denoted by $\Omega$, but in our case this set is finite.

Furthermore, there are also other variations between the classic bin packing problem and our problem. First, in our case, the capacities of channels are not homogeneous which means the capacity of the bins is not the same as in the classic problem. Second, unlike the bin packing problem, we do not consider minimizing cost incurred for channel or bin selection but instead we aim at maximizing the number of packed items in a finite number of bins (channels). This corresponds to maximize the achieved rate with the difference that even if the item does not fit into any bin, some part of the item can be packed in the bin. Finally, to consider the multi-channel communications, the volume of items or rate requirement of user $r_{j}^{q}$ can be divided into $n_{j}$ fragments which corresponds to the number of antennas. Consequently, these fragments of rate requirement must be assigned to the eligible channels considering their available capacity and the number of wireless interfaces.

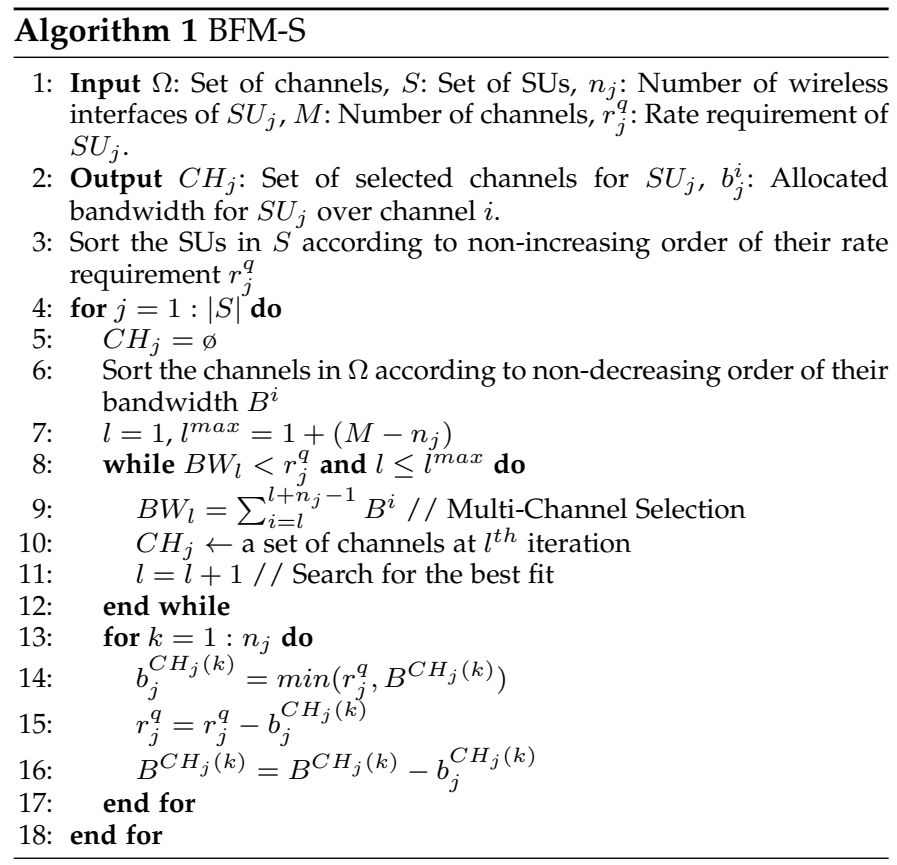

According to these variations, we adapt the BFD algorithm and propose Best Fit selection with Multi-channel constraint for Static spectrum sharing (BFM-S) heuristic to solve the spectrum sharing problem in hand. Pseudocode for the BFM-S algorithm is depicted in Algorithm 1. The details of the BFM-S algorithm are described as follows:

Multi-Channel Selection: Regarding utilization and fairness, the capacity of the selected channels should fit the rate requirement $r_{j}^{q}$. Otherwise, if this channel selection condition is not considered, any small value of $r_{j}^{q}$ can reserve a large bandwidth channel. This leads to inefficiencies in channel selection, larger request values of $r_{j}^{q}$ would have a small probability of being fulfilled. Thus, a large channel bandwidth should be reserved for SUs with large value of $r_{j}^{q}$.

To select the eligible channels for each SU, we first sort channels in $\Omega$ in non-decreasing order of their average total available bandwidth $B^{i}$. Second, we sort SUs in $S$ in non-increasing order regarding their rate requirement $r_{j}^{q}$. Accordingly, the BFM-S algorithm starts channel selection from the SU who has the maximum $r_{j}^{q}$. Fig. 2 illustrates the channel selection process for an SU. Here, the number of candidate channels in each iteration is restricted to the number of wireless interfaces $n_{j}$. Let $B W_{l}$ be the total bandwidth of a set of candidate channels at the $l^{t h}$ iteration, $B W_{l}=\sum_{i=l}^{l+n_{j}-1} B^{i}$ (line 9:). If the long-term bandwidth of candidate channels fits the rate requirement $r_{j}^{q}$, the iteration is broken and then the channels at this iteration are chosen for $S U_{j}$. A set of chosen channels for $S U_{j}$ is denoted by $C H_{j}$. This condition corresponds to $B W_{l} \geq r_{j}^{q}$ (line 8:). Note that the number of channels in our problem is finite and is denoted by $M$. Therefore, the number of iterations in the multi-channel selection is also finite and is calculated based on the number of antennas $n_{j}$ and the number of channels $M$. It is equal to $l^{\max }=1+\left(M-n_{j}\right)$. As a consequence, if an iteration runs until $l^{\max }$ and the rate requirement $r_{j}^{q}$ is larger than the long-term bandwidth of candidate channels $B W_{l}$, a set of channels at $l^{\max }$ iteration will be selected for $S U_{j}$.

Intra Channel Allocation: In this step, an SU is allocated a rate from each selected channel in $\mathrm{CH}_{j}$ which is obtained from the previous step. To allocate the bandwidth properly, first, the BFM-S algorithm assigns the channel that has the minimum remaining capacity to allocate the rate to $S U_{j}$. Consequently, the allocated rate for $S U_{j}$ over a selected channel $i$ is computed as: $b_{j}^{i}=\min \left(r_{j}^{q}, B^{i}\right), i \in C H_{j}$ (line 14:). If the channel does not have enough capacity to fit the $r_{j}^{q}$, the remaining rate requirement $\left(r_{j}^{q}-b_{j}^{i}\right)$ will be assigned to the next larger bandwidth channel in $\mathrm{CH}_{j}$ (line 15:). Finally, we update the bandwidth of the channels to the remaining bandwidth of the selected channels according to the allocated bandwidth $b_{j}^{i}$ (line 16:).

Now, the achieved rate of $S U_{j}$ can be computed as $a_{j}=\sum_{i=1}^{M} b_{j}^{i}$. This procedure is repeated for all users in the set $S$. Note that, when the algorithm starts processing the next user, the set of channels $\Omega$ needs to be sorted again because the bandwidth of some channels were changed after allocation of previous SUs.

When the total demand bandwidth of SUs is larger than the network capacity (overloaded state), the resource allocation may not be fair for all SUs, since some SUs may occupy 


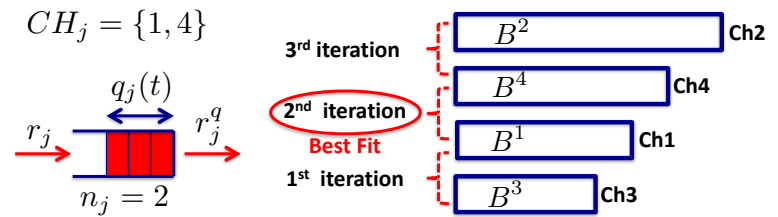

Fig. 2: An example of proposed BFM-S algorithm, SU equips with two antennas

all available bandwidth and the others will not be able to obtain any rate, especially the last one in set $S$. In fact, the allocation in SSS is applied for long-term transmission, as a consequence SUs who cannot satisfy the allocated rate will suffer from this insufficient rate allocation for a long period. Thus, the second step BFM-S is proposed to alleviate this unfairness problem. Note that this step is only applied in the case where at least one $\mathrm{SU}$ is not satisfied with its achieved rate $\left(a_{j}<r_{j}^{q}, \forall j \in\{1,2, \ldots N\}\right)$. In this step, the rate requirement $r_{j}^{q}$ is recomputed based on the total amount of allocated rate from the first step allocation, denoted by $A^{1 s t}=\sum_{j=1}^{N} a_{j}$. This amount represents somewhat the effective maximum bandwidth of the channels. Accordingly, rate requirements of SUs in the second step can be computed as follows:

$$
r_{j}^{q^{2 n d}}=\frac{r_{j}^{q}}{\sum_{j=1}^{N} r_{j}^{q}} \cdot A^{1 s t}
$$

Consequently, the BFM-S is recalled by applying the second step rate requirement $r_{j}^{q^{2 n d}}$. On the contrary, this unfairness problem cannot be solved at the first step of BFM$S$ through the classic weighted fair sharing method [26], due to the fact that the efficiency of the algorithm itself is also dependent on the number of wireless interfaces of each SU. Specifically, even in the underloaded state, rate requirements of SUs cannot be guaranteed because of the limitation of wireless interfaces, as described in Section 2, and thus the first step is necessary. Intuitively, the second step BFM-S enhances the fairness allocation, but it possibly decreases the utilization of channel bandwidth, since the maximum achieved rate is limited to the $r_{j}^{q^{2} n d}$. Therefore, we evaluate the first step and the second step BFM-S through the average service satisfaction, denoted by $E\left[\alpha_{j}\right]=\frac{\sum_{j=1}^{N} a_{j} / r_{j}}{N}$. If $E\left[\alpha_{j}\right]$ of the first step is larger than the second step, the allocation applies the solution from the first step BFM-S. Recall that, if all SUs can satisfy the rate requirement at the first step, the second step BFM-S is not necessary.

\subsection{Dynamic Spectrum Sharing}

Intuitively, when the rate requirement is larger than the available bandwidth of the channel, spectrum handoff is necessary by switching from currently unavailable channels to the available ones. On the other hand, even in the DSS scheme, waiting at the current unavailable channel in an attempt to compensate later the lost rate is also useful since it reduces the number of spectrum handoffs, which in turn can increase the achieved rate. To balance this tradeoff, the DSS scheme uses a rate compensation approach by introducing a reservation period, denoted by $T_{r}$.

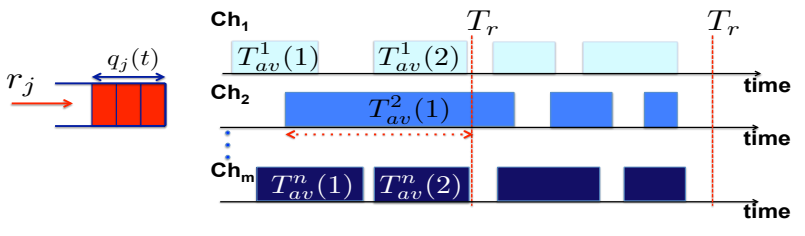

Fig. 3: The reservation period

A fixed allocation will be applied during this period without any handoff. If some SUs do not achieve their rate, they can compensate in the next reservation period by allocating to them a larger rate so that the average rate at the end of their connection meets the requirement. The allocation can change only at the end of each period as illustrated in Fig. 3. Thus, during unavailable periods inside $T_{r}$, spectrum handoffs are not performed. At the end of $T_{r}$, some SUs may not receive a sufficient bandwidth to send all their packets. This is reflected in their transmission queue $q_{j}(t)$. Thus, the requirement of SUs should be related to the number of packets in the queue in order to compensate the lost rate during the former $T_{r}$. The total quantity of data to be sent in the next period $T_{r}$ can be obtained as follows:

$$
p_{j}=r_{j} \cdot T_{r}+\sum_{q=1}^{q_{j}(t)} \operatorname{size}(q)
$$

where $q_{j}(t)$ is the number of packets in the transmission queue and size $(q)$ is the size of the $q^{\text {th }}$ packet in the queue. Unlike SSS, here the total capacity $c^{i}$ is computed through the quantity of data that the channel $i$ can "contain" in the next period $T_{r}$.

The information of channel availability plays an important role in DSS scheme. Several methods are presented in the prior work, for instance the CR base station can extract the channel availability from the regional databases as mentioned in RFC 7545 [27]. On the other hand, the CR base station can apply several predictive models based on the past channel usage which effectively avoid the interferences to PUs [16], [28], [29]. In this work, we rather focus on the spectrum sharing and handoff algorithms while assuming the information of channel availability is already been provided.

The quantity of data $c^{i}$ available for all users during $T_{r}$ is computed as follows:

$$
c^{i}=\sum_{l=1}^{k} T_{a v}^{i}(l) \cdot b w^{i}, \forall i \in\{1,2, \ldots M\}
$$

where $T_{a v}^{i}(l)$ is the $l^{t h}$ available period of channel $i$ in $T_{r}$, $k$ is the number of available periods in $T_{r}$ and $b w^{i}$ is the link bandwidth of channel $i$. To consider the overhead of spectrum handoff, the handoff delay $H O_{\text {delay }}$ is included if a channel was not selected in the former period. Thus, the capacity of channel $i$, when it is selected to $S U_{j}$ can be individually updated as follows:

$$
c_{j}^{i}=c^{i}-\left(\frac{\sum_{l=1}^{h} T_{a v}^{i}(l)}{\sum_{l=1}^{k} T_{a v}^{i}(l)} \cdot c^{i}\right), \text { if } i \notin C H_{j}^{f}
$$


where $h$ is the number of available periods during the handoff $\left(H O_{\text {delay }}\right)$ and $\mathrm{CH}_{j}^{f}$ is a set of selected channels in the former period. The last term on the right in the formula represents the fraction of data that is not used during the handoff. Note that, if $S U_{j}$ selects the same channel from the current set of active channels, i.e. $i \in C H_{j}$, the handoff delay is not counted for the channel capacity and $c_{j}^{i}=c^{i}$.

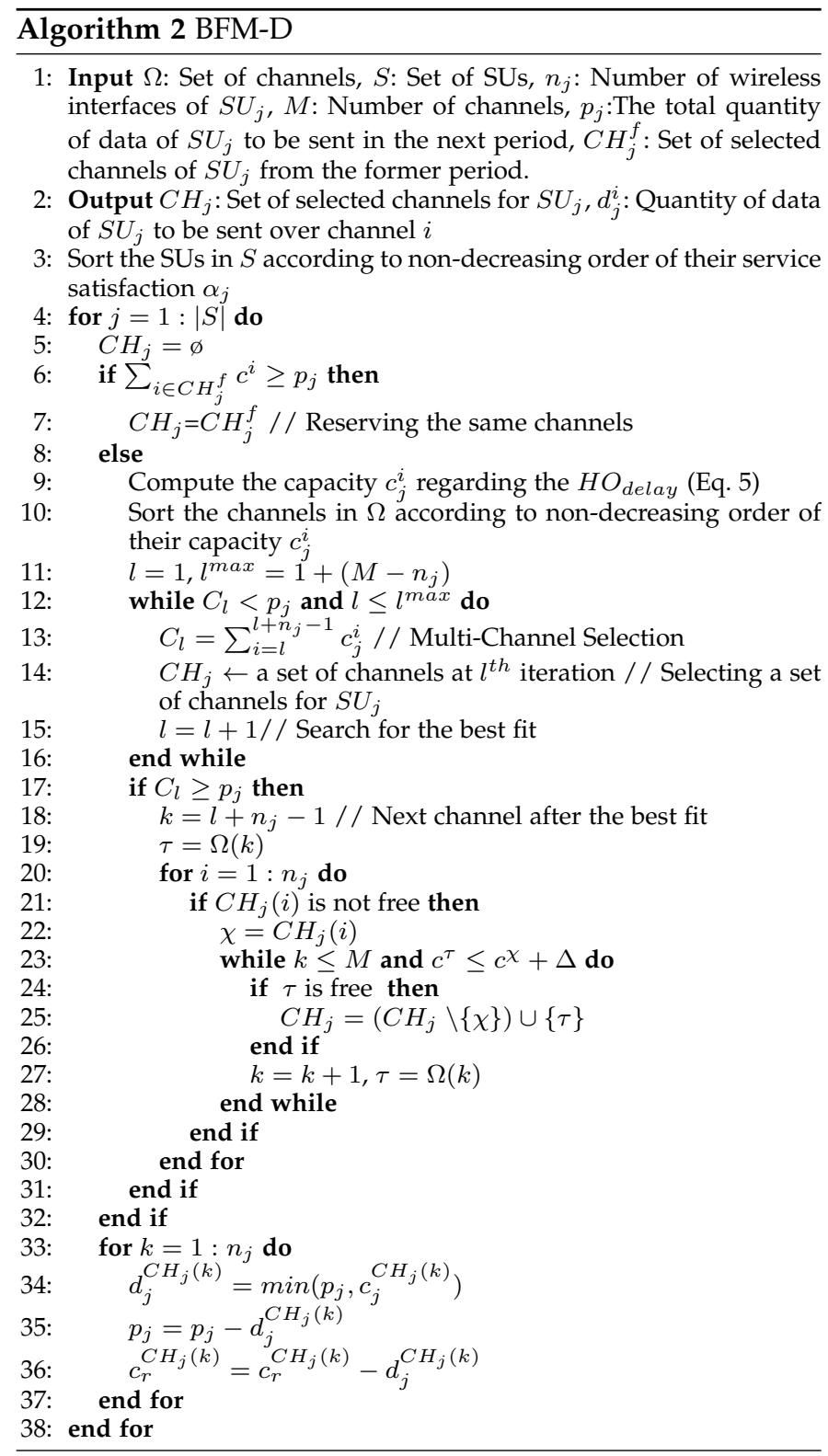

Similar to SSS, the BFM algorithm is also applied in DSS with some modifications, called Best Fit selection with Multi-channel constraint for Dynamic spectrum sharing (BFM$D)$. Pseudocode for the BFM-D algorithm is depicted in Algorithm 2. BFM-D is applied at the end of every $T_{r}$ period using $p_{j}$ and $c^{i}$ computed for the next $T_{r}$ period. As mentioned before, DSS applies a priority channel selection to compensate for insufficient resource allocation and to alleviate the unfairness problem. Therefore, the service satisfaction $\alpha_{j}$ is measured as the ratio between the achieved rate $a_{j}$ and the rate requirement $r_{j}$, which can be expressed as $\alpha_{j}=\frac{a_{j}}{r_{j}}, \forall j \in\{1,2, \ldots N\}$.
We sort SUs in $S$ in non-decreasing order according to their service satisfaction $\alpha_{j}$ (line 3:). Accordingly, the BFM$\mathrm{D}$ algorithm starts channel selection from the SU who has the minimum $\alpha_{j}$, in other words the most suffering SU who least satisfied its rate requirement. The capacity of channel $c^{i}$ must be updated according to the quantity of data that is allocated to the prior SUs. This updated capacity is called the remaining channel capacity, denoted by $c_{r}^{i}$. In addition, BFM-D also needs to determine the handoff delay for each SU individually (Eq. 5). However, the total channel capacity $c^{i}$ in the formula must be replaced by the remaining capacity $c_{r}^{i}$ because the channel capacity can be allocated to the higher priority SUs. Hence, before starting the selection for each SU, the set of $\Omega$ is sorted in non-decreasing order according to the remaining capacity $c_{j}^{i}$ which also includes the overhead of the handoff delay. (line 10:). The BFM$\mathrm{D}$ algorithm uses the total quantity of data $p_{j}$ instead of the rate requirement $r_{j}^{q}$. Therefore, the best fit condition is changed by considering $C_{l} \geq p_{j}$ (line 12:), where $C_{l}$ is the total capacity of a set of candidate channels.

However, in an overloaded state where the remaining available capacity in $T_{r}$ is less than the total rate requirements $\left(\sum_{i=1}^{M} c^{i}<\sum_{j=1}^{N} r_{j}\right)$, the resource allocation may not be fair, since the higher priority SU can monopolize channels with large available bandwidth, and the lower priority SUs cannot find any channels to maintain their rate requirements. On the one hand, some SUs can occupy all the available resources, but on the other hand, the others cannot obtain any transmission from the allocation. The SUs who are suffering from the unfair allocation have to wait until the next $T_{r}$ in order to get higher priority to occupy the limited available resources. Obviously, this causes some delays in the transmission and thus some burstiness which is not acceptable for some applications. To solve this issue, we compute the maximum allocation to limit the capacity that SU can obtain during the next reservation period. The maximum allocation of each SU can be computed based on the rate requirement and the total capacity of all channels, which can be expressed as follows:

$$
p_{j}^{\max }=\frac{r_{j}}{\sum_{j=1}^{N} r_{j}} \cdot\left(\sum_{i=1}^{M} \sum_{l=1}^{k} T_{a v}^{i}(l) \cdot b w^{i}\right)
$$

where $\left(\sum_{i=1}^{M} \sum_{l=1}^{k} T_{a v}(l) \cdot b w^{i}\right)$ is the total capacity of all channels in the next $T_{r}$ period. Note that, in the overloaded state, where the total rate requirement of SUs is larger than the network capacity, the demand for sending packets must be updated as: $p_{j}=p_{j}^{\max }$.

At the end of the reservation period $T_{r}$ a new allocation is recomputed, and some spectrum handoffs can be performed unnecessarily. To avoid generating unnecessary spectrum handoff at the end of the reservation period and thus further decreasing the number of spectrum handoffs, ${ }^{2}$ we incorporate two new mechanisms in the BFM-D algorithm as follows:

Reserving the same channels: During the multi-channel selection of BFM-D when the turn of $S U_{j}$ comes, then if the set

2. Recall that inside the reservation period $T_{r}$, spectrum handoffs are expected to be reduced since during unavailable periods the allocated channels are not changed. 
of current channels $C H_{j}$ used by $S U_{j}$ has sufficient capacity compared to $p_{j}$, the same set of channels is assigned to $S U_{j}$ as the former period (line 7:). If these channels were allocated to a previous user in the previous iterations, then $S U_{j}$ cannot keep the same channels. This is consistent with the fact that in the previous iterations higher priority (lesser satisfied) users were served first.

Avoiding taking a channel occupied by other SUs: The set of candidate channels $\mathrm{CH}_{j}$ would be preferably free from other SUs during the former period, due to the fact that other SUs may continue to use the same channels and thus spectrum handoff operation can be reduced globally. Therefore, the channel selection is continued to search for a new channel to replace an occupied channel in the best fit iteration. Clearly, the new replaced channel must be unoccupied by any SUs from the previous period. In addition, the capacity of this channel cannot be much larger than the one in the best fit iteration. Regarding the utilization aspect, an SU should be assigned to the first channel which fits its request. Otherwise, if an SU moves to the upper channel which has very large capacity, another SU who has a larger requirement will not be able to find any channel to fit its request. Here again, we must preserve the best fit principle. The criterion for selecting a new channel in a next step iteration to replace an occupied channel can be expressed as $c^{\tau} \leq c^{\chi}+\Delta$, where $\chi$ is an occupied channel found at the best fit iteration, $c^{\chi}$ is the capacity of channel $\chi, \Delta$ is a maximum bound capacity and $\tau$ is a free candidate channel from the next iterations that can replace the occupied channel in the best fit iteration. Accordingly, if channel $\tau$ is found within the maximum iteration $l^{\text {max }}$, the set of candidate channels from the best fit iteration is updated as follows: $\mathrm{CH}_{j}=\left(\mathrm{CH}_{j} \backslash\{\chi\}\right) \cup\{\tau\}$, (line 25:). However, if there is no channel that has this criterion, the algorithm will choose the occupied channel from the best fit iteration as originally found. Here, $\Delta$ is chosen to be small in order not to affect the best-fit principle. This mechanism is drawn in the line 20 - 30:.

Because $p_{j}$ is used instead of $r_{j}^{q}$, we then compute the quantity of data of $S U_{j}$ to be sent over channel $i$, which can be expressed as follows: $d_{j}^{i}=\min \left(p_{j}, c_{j}^{i}\right), \forall i \in C H_{j}$, (line 34:). The allocated bandwidth $b_{j}^{i}$ can be simply calculated by dividing $d_{j}^{i}$ by the total used available periods in the next $T_{r}$.

Consequently, the BFM-D algorithm also updates the capacity of the channels to the remaining capacity of the selected channels regarding the quantity of data $d_{j}^{i}$, which can be calculated as; $c_{r}^{i}=c_{r}^{i}-d_{j}^{i}, \forall i \in C H_{j}$, (line 36:). This refers to the intra channel allocation which is presented in the line 33 - 37:. Fig. 4 illustrates the procedures in BFM-D algorithm. The channels in $\Omega$ are sorted in non-decreasing order according to their remaining capacity $c_{r}^{i}$. Here, the best fit is found at the $2^{\text {nd }}$ iteration which contains channel 1 and 4 . However, channel 4 was occupied by the other SUs in the previous $T_{r}$ period $(\chi=4)$, hence the iteration is continued to search for an appropriate channel $(\tau)$. At the next iteration, there is channel 2 which was not occupied by any $\mathrm{SU}$ from the previous $T_{r}$. Besides, its capacity is also not much larger than channel $4\left(c_{r}^{2} \leq c^{\chi}+\Delta\right)$. As a consequence, channel 2 is chosen instead of channel 4 (i.e. $C H_{j}=\{1,2\}$ ).
Finally, BFM-D allocates the quantity of data $d_{j}^{1}$ and $d_{j}^{2}$ over channel 1 and 2 respectively.

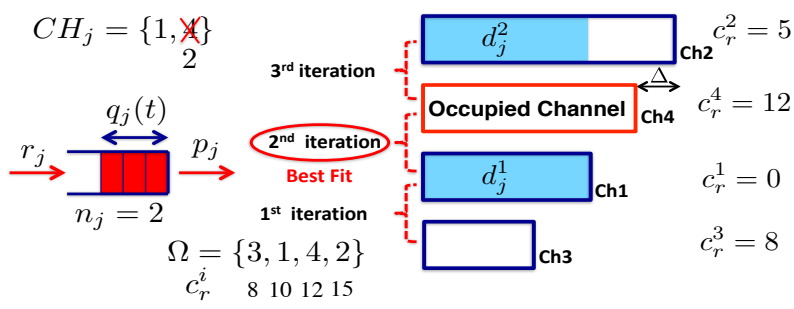

Fig. 4: An example of an allocation in BFM-D algorithm, SU equipped with two wireless interfaces

\subsection{Hybrid Spectrum Sharing Decision}

According to the dynamic PU activities, the environment of CR networks varies over time, which makes it more difficult to decide on a spectrum sharing scheme while maintaining the service requirements and reducing the number of spectrum handoffs. Besides, both SSS and DSS can provide good performance in different network configurations. However, to decide by only one sharing mode is not sufficient due to the dynamic environment in CR networks where various configurations can be found during the communication of SUs. Therefore, it is preferable to apply a hybrid decision in which the mode of spectrum sharing can be interchangeable between SSS and DSS. The state diagram for the hybrid decision is shown in Fig. 5.

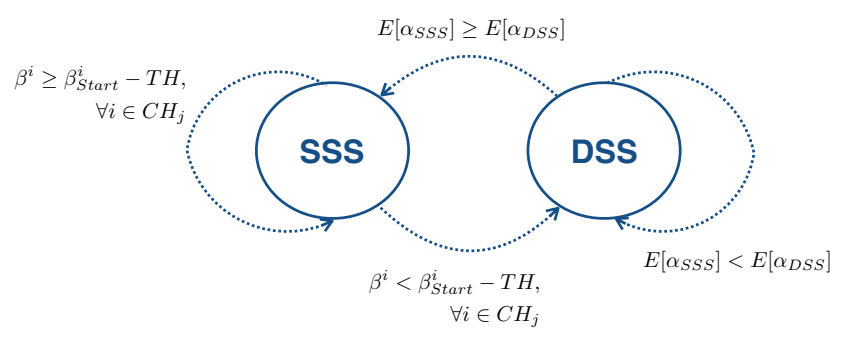

Fig. 5: The two modes of the hybrid spectrum sharing

Clearly, SSS is more suitable for the underloaded state where the rate requirements are less than the capacities of the channels. However, it is not sufficient to choose SSS based solely on this condition. Due to the limitation of the number of wireless interfaces, all SUs may not satisfy their rate requirement even though the network is classified as underloaded. On the other hand, applying only the DSS to achieve a higher rate may not be necessary, since a lot of spectrum handoff would be performed. To make the right decision between SSS and DSS, at the beginning of each $T_{r}$ period, we apply the following procedures and rules:

Current spectrum sharing is DSS: Firstly, SSS is run to find a solution for resource allocation and to estimate the average satisfaction, $E\left[\alpha_{S S S}\right]$. Secondly, the average satisfaction of SSS is compared to the current measured one, $E\left[\alpha_{D S S}\right]$. Finally, if the satisfaction from SSS is better than the current 
one (DSS), the spectrum sharing is switched to SSS mode. Otherwise, spectrum sharing is still in DSS scheme and BFM-D is called to find a solution for spectrum sharing in the next $T_{r}$ period.

Current spectrum sharing is SSS: The achieved rate of SUs is sensitive to the availability ratio of channel, denoted by $\beta^{i}$. Since the BFM-S algorithm requires this ratio to estimate the capacity of channels, it is important to observe this characteristic of channels regularly. Moreover, if this characteristic is fluctuating over some acceptable limit, BFM-S is recalled to compute a new solution for spectrum sharing. This means that the initial allocation of SSS cannot provide the guaranteed service satisfaction to all SUs. The initial availability ratio of a channel, denoted by $\beta_{\text {Start }}^{i}$ is recorded when spectrum sharing is switched from DSS to SSS. Furthermore, the available and unavailable periods have to be measured in a long-term observation period, for instance from the current time back to the instant when the transmission started. This long-term observation is useful, since it can capture all characteristics of a channel including the fluctuation of the available periods. Consequently, $\beta^{i}$ is computed at the beginning of each $T_{r}$ based on the average of the available and unavailable periods from the long-term observation. Finally, if at least one active channel (a channel used by SU) has $\beta^{i}$ below the $\beta_{\text {Start }}^{i}$, the spectrum sharing will switch to DSS and the BFM-D is called to find a solution for spectrum sharing in the next $T_{r}$ period. The exact condition to move from SSS to DSS is: if $i \in C H_{j}$ such that $\beta^{i}<\beta_{\text {Start }}^{i}-T H . T H$ is the acceptable limit, which should be small. Besides, the value $T H$ can be guided by the application; for instance, the file transfer protocol may accept a small tolerance allowing a reduction in the sending rate which can be related to the acceptable limit of the availability ratio.

\subsection{Complexity Analysis}

BFM-S algorithm contains three procedures including the call to sort (line 3: and 6:), multi-channel selection (line 812:) and intra channel allocation (line 13- 17:). Concretely, sorting a set of channels has a complexity of $O(M \log (M))$, where $M$ is the number of licensed channels. Then, the multi-channel selection procedure requires a number of executions in the order of $l^{\max }$ which corresponds to the number of antennas $n_{j}$ and the number of channels $M$. The maximum number of iterations can be equal to $M$ and thus the time complexity of this procedure is upper bounded by $O(M)$. The intra channel allocation is bounded by the number of selected channel $n_{j}$ which, in the worst case, is $M$. Clearly, this procedure also implies a complexity of $O(M)$. In our case, BFM-S is applied for all $N$ SUs, hence the BFM-S algorithm can be carried out in polynomial time $O(N M \log (M))$, since the running time is dominated by the sorting procedure.

Similar to BFM-S algorithm, sorting, multi-channel selection and intra channel allocation procedures are also integrated in the BFM-D algorithm. Apart from those procedures, BFM-D introduces a new procedure that avoids to take an occupied channel by other SUs (line 20 - 30:). When the best fit selection is found, BFM-D algorithm necessitates afterwards checking the occupancy of the selected channels in $\mathrm{CH}_{j}$ and replacing occupied channels by free channels from the next iterations. In the worst case, BFM-D may select all occupied channels to $S U_{j}$ from the first iteration $(l=1)$, then $k=n_{j}$ (line 18:). All these occupied channels can be replaced by the unoccupied channels from the next iteration. The number of iterations can be varied from $k$ to $M$ (line 23:), hence the complexity of this replacement process becomes $O(M)$. Note that this replacement process is not imbricated inside other procedures, thus the total complexity of BFM-D is then $O(N(2 M \log (M)+3 M))$. Clearly the BFM-D algorithm still runs in time $O(N M \log (M))$ as BFMS.

At the beginning of each reservation period $T_{r}$, the hybrid spectrum sharing heuristic needs to decide the mode of spectrum sharing: either SSS or DSS. If the current spectrum sharing mode is SSS, the availability ratio of the channel or $\beta^{i}$ must be computed for all $M$ channels to estimate the channel quality. If the hybrid decides to move from SSS to DSS, the BFM-D is called. Thus the complexity of this decision is upper bounded by $O(M+N M \log (M))$. On the other hand, when the current spectrum sharing is DSS, the BFM-S is called first to estimate $E\left[\alpha_{S S S}\right]$ and compared with the one from the current measurement. In the worst case, the hybrid decision decides to stay on the DSS scheme, and thus the BFM-D is applied to find a solution for the next $T_{r}$. Concretely, the time complexity of this decision includes both BFM-S and BFM-D which still corresponds to $O(N M \log (M))$.

\section{Performance Evaluation}

In this section, we analyze the performance of our proposed hybrid spectrum sharing strategy using OMNeT++ [30]. The performance of our strategy is compared to dynamic spectrum sharing where the spectrum sharing is based solely on DSS. Furthermore, we also compare our hybrid strategy with the Global Spectrum Sharing (GSH) [22]. In this model, global knowledge of PUs' activities is assumed to be available in advance. The optimization problem in GSH is solved by programming using CPLEX 12.4 [31]. The simulation is repeated 20 times, or 30 if confidence intervals are very large. Averages along with $95 \%$ confidence intervals computed with the t-distribution, are shown in all plots. In addition, the simulation time is fixed to 500 time units.

\subsection{Simulation Setup and Performance Metrics}

We simulate an infrastructure-based CRN with $M=12$ licensed channels and a total of $N=4$ secondary users. Without loss of generality, each channel has a fixed bandwidth of 10 packets/time unit $\left(b w^{i}=10\right)$ and the size of a transmission packet is fixed as 1 unit for all SUs. To take into account the PUs' activities, we simulate channels that are switching between available and unavailable periods with a duration that is exponentially distributed. The means of these durations, denoted by $E\left[T_{a v}\right]$ and $E\left[T_{u n}\right]$, are generated between $\{0.33,4.5\}$ time units in order to create 12 channels with different properties (Table 2). We compare the performance of the spectrum sharing strategies while increasing the SU's rate requirement. At the first step, 4 
SUs have different rate requirements equal to $1,2,3$ and 4 respectively, hence the total rate requirement $(R)$ is equal to 10 . Then, we increase the rate requirement of each $\mathrm{SU}$ from the first step by $\{2,3,4,5,6,7,8,9,10\}$ times, thus the total rate requirement is varied as follows: $10,20,30,40,50$, $60,70,80,90$ and 100 successively.

TABLE 2: Channel configuration with exponential distribution

\begin{tabular}{cccc|cccc}
\hline$C h$ & $E\left[T_{a v}\right]$ & $E\left[T_{u n}\right]$ & $\beta^{i}$ & $C h$ & $E\left[T_{a v}\right]$ & $E\left[T_{u n}\right]$ & $\beta^{i}$ \\
\hline 0 & 1 & 3 & 0.25 & 6 & 1.5 & 1.5 & 0.5 \\
1 & 1.5 & 4.5 & 0.25 & 7 & 2 & 2 & 0.5 \\
2 & 2 & 6 & 0.25 & 8 & 1 & 0.33 & 0.75 \\
3 & 4 & 12 & 0.25 & 9 & 1.5 & 0.5 & 0.75 \\
4 & 0.5 & 0.5 & 0.5 & 10 & 2 & 0.67 & 0.75 \\
5 & 1 & 1 & 0.5 & 11 & 4 & 1.33 & 0.75 \\
\hline
\end{tabular}

To analyze the performance of the hybrid spectrum sharing strategy, we base our evaluation on total achieved rate, performed number of spectrum handoffs and the degree of fairness. Regarding the objective of achieving a fair spectrum sharing, the Jain's fairness index [32] is computed and compared with different total rate requirements $R$. So far, in our hybrid spectrum sharing, some input parameters are required in the configuration, consisting of the compensation period $T_{\text {comp }}$, maximum bound capacity $\Delta$, threshold of channel fluctuation $T H$ and the reservation period $T_{r}$. Clearly, the value of $T_{\text {comp }}$ should be large regarding the long-term communication in SSS. Thus, we set $T_{\text {comp }}=100$ time units and applied it for all scenarios. For the $\Delta$ parameter, clearly this value should be small, because we want to maximize the utilization of resource allocation, as we discussed amongst the details of the BFM$\mathrm{D}$ algorithm. Hence, we applied the value of 0.05 for $\Delta$. Similarly for the parameter $T H$, this value should be small as well, so spectrum sharing can switch to DSS faster. Hence, the value of $T H$ is set to 0.1 . The impact of the reservation period $T_{r}$ is crucial for our strategy, hence we study this factor precisely in the following section. In addition, we first omit the impact of handoff delay by setting $H O_{\text {delay }}=0$ in order to understand clearly the performance of hybrid spectrum sharing strategy. Then, we investigate this factor closely through section 4.6.

\subsection{Impact of Reservation Period}

We investigate first what would be the impact of the reservation period $\left(T_{r}\right)$. Fig. 6 plots the total achieved rates at the receivers, the number of performed handoffs during the simulation and fairness index of allocation while varying the value of $T_{r}$ and the total rate requirement of SUs, $R=\{20,40,60,80,100\}$. As mentioned before, the number of spectrum handoffs and the ability of rate compensation can be controlled through $T_{r}$. The results show that when $T_{r}$ is increased, the allocation is closer to the static allocation where the number of channel handoffs is significantly decreased. However, the total achieved rate is also decreased when $T_{r}$ is large, since SU cannot enhance the achieved rate by performing spectrum handoffs. On the contrary, the small reservation period $\left(T_{r}=0.25,0.5,1\right)$ also cannot enhance the achieved rates due to the fact that it is too short to allow a rate compensation to be set up. Indeed, we approach sharing with instantaneous handoffs without rate compensation. Furthermore, unnecessary handoffs are performed, since new allocation is recomputed periodically in each $T_{r}$ period. According to the figure 6 , a value of reservation period equals to two units of time achieves the best performance in terms of achieved rate and fairness with a low number of channel handoffs. Therefore, for the next simulations, we fix the reservation period to two units of time.

\subsection{Achieved Rates vs. Number of Handoffs}

In this section, the performance of hybrid spectrum sharing is studied and compared to the performance of dynamic spectrum sharing and GSH while varying the total rate requirements $(R)$ from 10 to 100 . Fig. 7 shows the summation of achieved rates for all SUs and compares it to the number of channel handoffs. Generally, GSH obtains slightly higher rates compared to the hybrid and dynamic strategies (see Fig. 7(a)). However, the GSH method performs excessively more handoffs than the others which is not efficient enough to be justified by the small gain in the rate it achieves. Fig. 7(b)) shows the efficiency of both BFM$S$ and BFM-D heuristics for the rate allocation. The reason for this efficiency is that the hybrid and dynamic strategies integrate many features to prevent unnecessary handoffs. These features are: reserving the same channel, avoiding taking channels occupied by other SUs, and not performing handoffs during a reservation period.

Both hybrid and dynamic strategies provide similar performance in terms of total achieved rates. However, in the underloaded state, i.e., when all SUs can satisfy their rate requirement $(R=10$ and 20$)$, the hybrid strategy shows a lower number of channel handoffs (see Fig. 7(b)). This is because the hybrid strategy switches to the SSS scheme where SUs can satisfy their allocation without any attempt to perform handoffs. On the other hand, in the very high load state ( $R=70$ onwards), the hybrid strategy efficiently performs handoffs leading to a lower number of channel handoffs, while it achieves almost the same total achieved rate as the dynamic strategy. Through spectrum sharing decision, it is sufficient to apply the static allocation in this environment, since the available bandwidth is scarce compared to the rate requirement. Thus, spectrum handoff is less necessary. Nevertheless, even though the average available bandwidth is 60 , all strategies (GSH, hybrid and dynamic) still cannot satisfy the total rate requirement when it is larger than 20 .

\subsection{Multi-Channel Benefits for Hybrid Sharing}

According to the limitation on the number of wireless interfaces, SUs may not be able to achieve their rate requirement and thus maximize the utilization of the spectrum. In this section, we consider that all SUs are equipped with the same number of wireless interfaces $n_{j}$ and the total rate requirement is set to $R=80$. Fig. 8 (a) shows the total achieved rate while the number of interfaces is varied from one to 12 . Using three wireless interfaces, the achieved rates can reach to the maximum utilization.

As for the number of handoffs (Fig. 8(b)), using $n_{j}=1,2$ wireless interfaces is not sufficient to provide more transmission opportunities to increase all users satisfactions. 


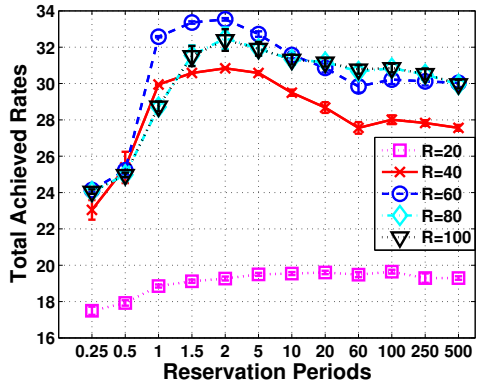

(a) Total achieved rates

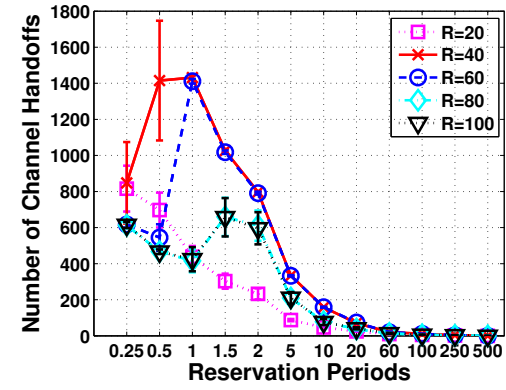

(b) Number of channel handoffs

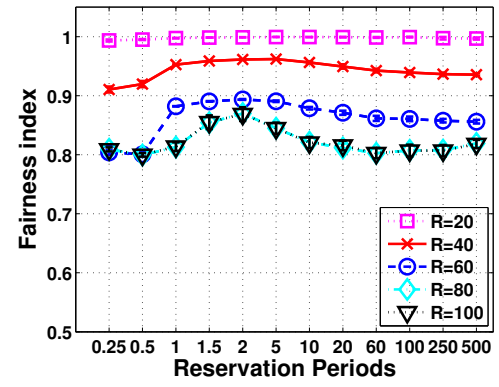

(c) Fairness index

Fig. 6: Impact of reservation period

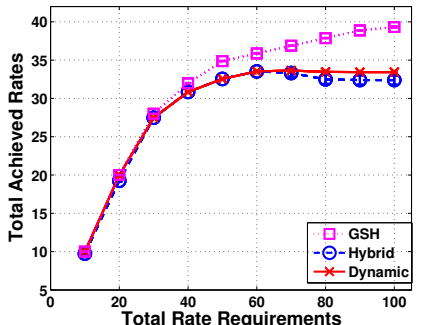

(a) Total achieved rates

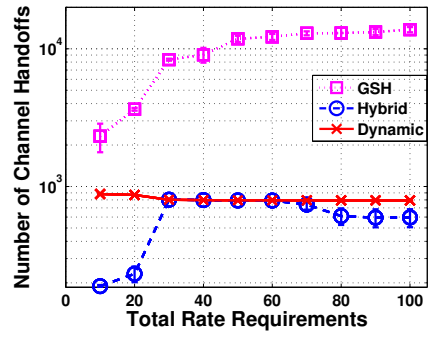

(b) Number of channel handoffs
Fig. 7: Performance for different spectrum sharing strategies

Hence, by increasing the number of wireless interfaces, spectrum handoff is more useful to have more satisfaction beyond the achieved rate. Consequently, the number of channel handoffs is significantly increased when a wireless interface is added, especially for dynamic spectrum sharing. On the other hand, when $n_{j}=3$ onwards, spectrum handoff is reduced significantly, showing that the limitation on the number of wireless interfaces is released. In fact, the total number of wireless interfaces in the network equals the total number of channels $(4 \times 3=12)$. To conclude, hybrid spectrum sharing strategy can reduce the number of channel handoffs significantly compared to the dynamic approach, since the former can switch to static mode which is very useful to avoid performing unnecessary spectrum handoff.

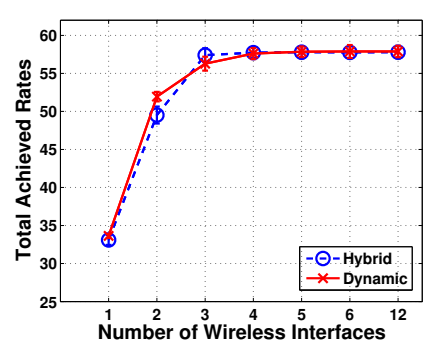

(a) Total achieved rates

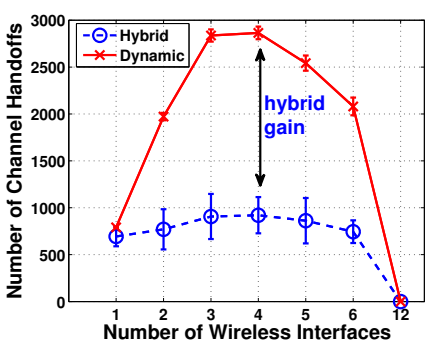

(b) Number of channel handoffs
Fig. 8: Impact of multi channel communications

\subsection{Impact on Fairness}

In this section, the Jain's fairness index [32] is computed and compared with different total rate requirements $R$ by fixing $n_{j}=1$ (see Fig. 9(a)). Apparently, the maximum fairness cannot be achieved from 20 onwards, because the available bandwidth can be supplied only to some SUs and not for all. Thus, the service satisfaction can be different among SUs, but the utilization is increased reasonably. However, when $R>60$, the fairness of dynamic strategy is slightly improved. This is because the system turns to the overloaded state and all SUs cannot satisfy the service requirements, leading to the global increase in fairness. Besides, the priority channel selection in the dynamic strategy (DSS scheme) allows SUs who achieve low service satisfaction to benefit from the higher priority to compensate for lost rates in turns and achieve fairness in the long term. Conversely the hybrid strategy shows lower fairness because it prefers to apply the static allocation (SSS scheme) to avoid performing a large number of spectrum handoffs. If some SUs cannot satisfy their requirements, they will not be able to achieve their satisfaction. Furthermore, multi channel communications also improve fairness, as shown in Fig. 9(b). The larger the number of wireless interfaces, the better the tradeoff between fairness and utilization. As a result, when the limitation on the number of wireless interfaces is released $\left(n_{j}=3\right)$, the maximum fair allocation is attained.

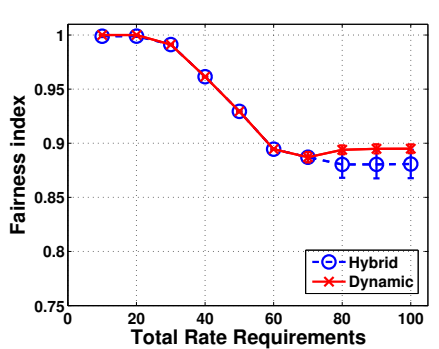

(a) Varying rate requirement

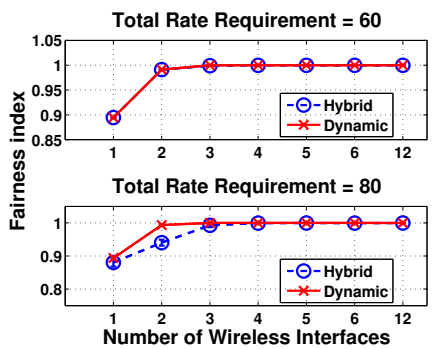

(b) Varying number of wireless interfaces
Fig. 9: Jain's fairness index of hybrid and dynamic spectrum sharing strategies

\subsection{Impact of Handoff Delay}

In this section, we vary the handoff delay from 0 to 1.5 units of time while the total rate requirement is set to $R=80$. In 
Fig. 10(a), we show that increasing handoff delay causes an adverse influence on the total achieved rate in both hybrid and dynamic strategies. Generally, the dynamic strategy can achieve better performance in terms of total achieved rate, but the number of channel handoffs is significantly increased (see Fig. 10(b)). On the contrary, when handoff delay is increased, the hybrid strategy achieves a better rate than the dynamic one. During the handoff process, the SU must stop its on-going transmission, and decides to either wait at the busy channel or switch to an idle one. This significantly impacts the achieved rate, especially when the handoff delay is large. Because of this, the the hybrid strategy decides effectively to apply the SSS scheme where the allocated channels are not changed. As a result, the handoff cost can be alleviated and the total achieved rate is also enhanced. Besides, the capacity of new target channels is reduced by the handoff delay, (Eq. 5) causing a reduction in the average satisfaction ratio of DSS, so that SSS becomes a better choice.

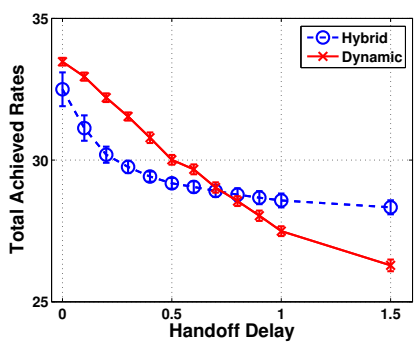

(a) Total achieved rates

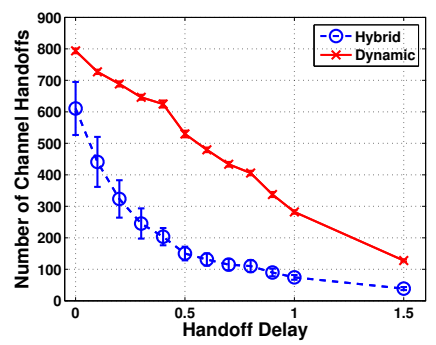

(b) Number of channel handoffs
Fig. 10: Impact of handoff delay

\subsection{Impact on High Variance of Availability Period}

We keep the on/off model for the activities of primary users over the licensed channels. However, the available and unavailable periods of the licensed channels are generated from the Pareto distribution. The probability density function of a Pareto random variable is expressed as follows:

$$
f_{X}(x)= \begin{cases}K \frac{(X m)^{K}}{x^{(K+1)}} & \text { for } x \geq X m, \\ 0, & \text { for } x<X m\end{cases}
$$

where $K$ is the tail index and $X m$ is the minimum possible value of $x$. We simulate 12 channels with different properties while keeping the same expected value of available and unavailable periods, $E\left[T_{a v}\right], E\left[T_{u n}\right]$, as applied in the previous section. Therefore, we fix $K=1.5$ and calculate the value of $X m$ based on the $E\left[T_{a v}\right], E\left[T_{u n}\right]$. Accordingly, the expected value of an available period following Pareto distribution can be computed as follows:

$$
E\left[T_{a v}\right]=\frac{K_{a v} \cdot X m_{a v}}{K_{a v}-1}, K_{a v}>1
$$

Without loss of generality, the expected value of an unavailable period can also follow the same formula by changing the subscript from $a v$ to $u n$. The channel configurations are mentioned in Table 3.

Similar to the previous section, we compare the performance between hybrid and dynamic spectrum sharing
TABLE 3: Channel configuration with Pareto distribution

\begin{tabular}{cccc|cccc}
\hline$C h$ & $X m_{a v}$ & $X m_{u n}$ & $\beta^{i}$ & $C h$ & $X m_{a v}$ & $X m_{u n}$ & $\beta^{i}$ \\
\hline 0 & 0.333 & 1 & 0.25 & 6 & 0.5 & 0.5 & 0.5 \\
1 & 0.5 & 1.5 & 0.25 & 7 & 0.667 & 0.667 & 0.5 \\
2 & 0.667 & 2 & 0.25 & 8 & 0.333 & 0.111 & 0.75 \\
3 & 1.333 & 4 & 0.25 & 9 & 0.5 & 0.167 & 0.75 \\
4 & 0.167 & 0.167 & 0.5 & 10 & 0.667 & 0.222 & 0.75 \\
5 & 0.333 & 0.333 & 0.5 & 11 & 1.333 & 0.444 & 0.75
\end{tabular}

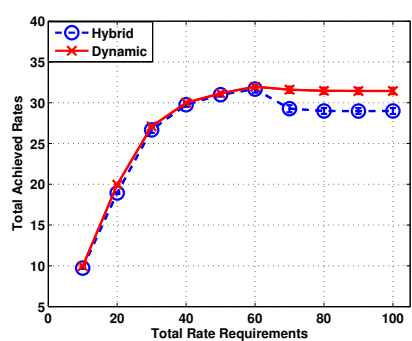

(a) Total achieved rates (b) Number of channel handoffs

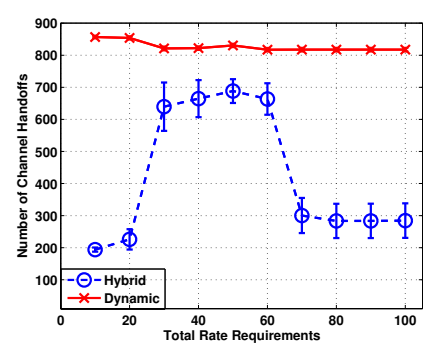

Fig. 11: Rate performance and number of channel handoffs: PUs' activities follow Pareto distribution

in terms of rate performance, number of channel handoffs and fairness. Fig. 11 shows the performance of rate achievements and the number of channel handoffs. Most of the results provide the same tendency with the exponential distribution. However, in the very high load state ( $R=70$ onwards), the hybrid strategy generates a very low number of channel handoffs which is even lower than the one in the exponential distribution case. This is because Pareto distribution is a power law probability distribution in which the generating random numbers fluctuate a great deal. Generally, available and unavailable periods can be very long or very short. Therefore, in the case where hybrid strategy switches to the static mode, most likely when it is in a long available period, the sharing strategy will not switch to dynamic mode. Obviously during this specific period, the number of spectrum handoffs can be reduced significantly compared to the exponential distribution case. On the contrary, when the available period is short, the hybrid strategy usually applies the dynamic mode in both Pareto and exponential distributions. Since the total rate requirement is high, spectrum handoff is necessary in order to enhance the achieved rate.

\subsection{Adaptivity to changing network parameters}

In this section, we evaluate the efficiency of hybrid spectrum sharing strategy by considering an abrupt change of PUs' activities parameters. The available/unavailable periods of channels 8 to 11 are switching between two exponential distributions in every 200 time units. Table. 4 illustrates the channel configurations with the two exponential distributions for channels 8 to 11 . The first 8 channels are the same as before (Table.2) without changing the distribution for the whole period of the simulation. To better emphasize the efficiency of the hybrid spectrum sharing strategy, we consider simulation time for 1000 time units. The rate requirement of SUs, $r_{1}$ to $r_{4}$, are set to $6,12,18$ and 24 respectively $(R=60)$. As for the other parameters, we keep the same configuration as section 4.3 (i.e., $T_{r}=2$, 
$\left.n_{j}=1, H O_{\text {delay }}=0, T_{\text {comp }}=100, b w^{i}=10\right)$. Another adequate behaviour of our hybrid sharing is that all SUs handoff to channel $8-11$ when the channels' configuration follows the second distribution to achieve the better rates. Indeed, during the first distribution, the total rate requirement $R$ is larger than the total average available bandwidth $\left(C=\sum_{i=1}^{M} \beta^{i} \cdot b w^{i}\right.$, i.e. $\left.C=40\right)$. In the second distribution, channels $8-11$ have a higher average availability ratio. As a consequence, the network becomes underloaded in the second phase $(C>R$, i.e., $C=62)$.

TABLE 4: Channel configurations with the two different exponential distributions

\begin{tabular}{l|lll|lll}
\hline Ch & \multicolumn{3}{|c|}{ First distribution } & \multicolumn{3}{c}{ Second distribution } \\
& $E\left[T_{a v}\right]$ & $E\left[T_{u n}\right]$ & $\beta^{i}$ & $E\left[T_{a v}\right]$ & $E\left[T_{u n}\right]$ & $\beta^{i}$ \\
\hline 8 & 1 & 3 & $\mathbf{0 . 2 5}$ & 1 & 0.25 & $\mathbf{0 . 8}$ \\
9 & 1.5 & 4.5 & $\mathbf{0 . 2 5}$ & 1.5 & 0.375 & $\mathbf{0 . 8}$ \\
10 & 2 & 6 & $\mathbf{0 . 2 5}$ & 2 & 0.5 & $\mathbf{0 . 8}$ \\
11 & 4 & 12 & $\mathbf{0 . 2 5}$ & 4 & 1 & $\mathbf{0 . 8}$ \\
\hline
\end{tabular}

Fig. 12 shows the operating scheme of spectrum sharing during the simulation. Accordingly, the spectrum sharing scheme is switched adaptively between SSS and DSS regarding the current network configuration and the service satisfaction of SUs. According to the channel configuration, during simulation time: 0-200, 400-600, the average available capacity of the channels is rather low where channels $8-11$ have a small value of availability ratio $\beta^{i}$. Therefore, our hybrid strategy decides to use the DSS scheme, since SUs can benefit from spectrum handoff to enhance the achieved rate. On the other hand, when we switch to the second distribution, the operating spectrum is switched to the SSS scheme after a transition period. This is because the average available capacity of the channels is increased.

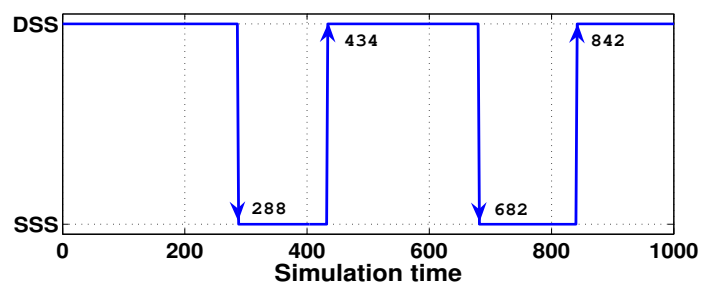

Fig. 12: Chosen spectrum sharing scheme over time

Interestingly, the hybrid strategy does not move to SSS immediately when the network configuration changes (simulation time $=201,601$ ). This is due to the fact that during this transition time SUs still have some packets buffered in the transmission queue. Apparently, spectrum handoff is still useful to switch from an unavailable channel to the available one, whereupon the buffered packets can be distributed from the transmission queue. Furthermore, the hybrid strategy requires some periods to ensure that the allocation of SSS is adequate for the rate requirements since SUs are not allowed to perform handoff in SSS. Thus, the transition time to switch to SSS is moderately long. On the other hand, when the channels' configuration switches back to the first distribution (simulation time $=401,801$ ), hybrid strategy decides effectively to switch to DSS, since the available bandwidth of the first distribution is not adequate for the rate requirements of all SUs. The transition time for switching from SSS to DSS must be short since SUs cannot benefit from spectrum handoff, leading to low rate performance. As a consequence, the performance of the hybrid strategy follows this property for the later simulation time. Moreover, we also compare the efficiency of the hybrid strategy with the dynamic one in terms of achieved rate and number of channel handoffs for each SU individually, as illustrated in Fig. 13. Obviously, the Hybrid strategy has a lower number of channel handoffs for all SUs, since the spectrum sharing is switched to the static allocation SSS. As for the rate performance, the hybrid strategy achieves a slightly lower rate than the dynamic strategy. These results show that our dynamic strategy with the BFM-D algorithm is able to maximize efficiently the utilization and the user satisfaction. However, our hybrid strategy still performs a better tradeoff between the number of spectrum handoffs and the achieved rates.

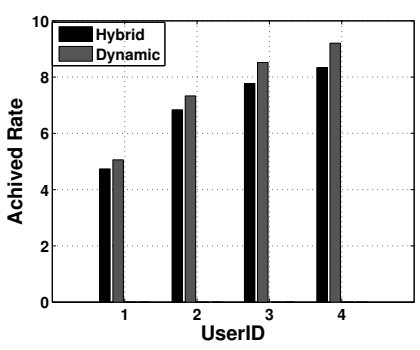

(a) Achieved rates per SU

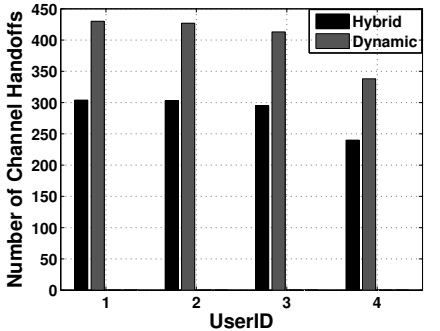

(b) Number of channel handoffs per SU
Fig. 13: The efficiency of hybrid spectrum sharing

\section{CONCLUSION}

In this work, we have proposed a hybrid spectrum sharing strategy which includes two heuristic algorithms: static spectrum sharing and dynamic spectrum sharing. These two sharing algorithms are selected adaptively depending on the current network status. We compare the performance of the hybrid strategy to that of the dynamic strategy the number of spectrum handoffs and the achieved rates where spectrum handoff is triggered periodically at the end of a reservation period. The simulation results shows that both the dynamic and the hybrid are able to fulfill the achieved rates thanks to multi-channel adapted best fit algorithms and the rate compensation concept. The hybrid strategy performs better in terms of tradeoff between maximizing the achieved rates and reducing the number of handoffs. An interesting finding is that using long-term future information like the global optimization (GSH) does not necessarily obtain the maximum rate performance. This is due to the fact that the long-term future information does not provide any clear insight on how to perform spectrum handoff, and the problem becomes more complex, since there are many possibilities for the spectrum handoff and selection decisions using this future information.

In future work, we will investigate the impact of spectrum sensing and how to provide an appropriate solution 
for the imperfect sensing information. Additionally, extending our proposed spectrum handoff and selection algorithms to distributed cognitive radio networks is a possible future research direction that requires a robust design for control information exchange.

\section{REFERENCES}

[1] W.-Y. Lee and I. F. Akyldiz, "A spectrum decision framework for cognitive radio networks," IEEE Transactions on Mobile Computing, Feb. 2011.

[2] D. Gozupek and F. Alagoz, "Throughput and delay optimal scheduling in cognitive radio networks under interference temperature constraints," Communications and Networks, Journal of, vol. 11, no. 2, pp. 148-156, 2009.

[3] L. Le and E. Hosain, "Resource allocation for spectrum underlay in cognitive radio networks," IEEE Transactions on Wireless Communications, Dec. 2008.

[4] D. Lu, X. Huang, W. Zhang, and J. Fan, "Interference-aware spectrum handover for cognitive radio networks," Wireless Communication and Mobile Computing, Sept. 2012.

[5] L. Akter and B. Natarajan, "Qos constrained resource allocation to secondary users in cognitive radio networks," Computer Communications, Dec. 2009.

[6] Y. Xing, C. Mathur, M. Haleem, R. Chandramouli, and K. P. Subbalakshmi, "Dynamic spectrum access with qos and interference temperature constraints," IEEE Transactions on Mobile Computing, Apr. 2007.

[7] J. Tang, S. Misra, and G. Xue, "Joint spectrum allocation and scheduling for fair spectrum sharing in cognitive radio wireless networks," Computer Networks, Aug. 2008.

[8] C. Peng, H. Zheng, and B. Y. Zhao, "Utilization and fairness in spectrum assignment for opportunistic spectrum access," Mobile Networks and Applications (MONET), Aug. 2006.

[9] H. Zheng and C. Peng, "Collaboration and fairness in opportunistic spectrum access," in Proc. IEEE ICC, 2005.

[10] T. Li, W. Tang, and S. Li, "A packet scheduling algorithm with interruption factor for cognitive radio," in IEEE ICIS, 2010.

[11] D. Xu, E. Jung, and X. Liu, "Efficient and fair bandwidth allocation in multichannel cognitive radio networks," IEEE Transactions on Mobile Computing, Aug. 2012.

[12] -, "Optimal bandwidth selection in multi-channel cognitive radio networks: How much is too much?" in Proc. IEEE DySPAN, Oct. 2008.

[13] A. Lertsinsrubtavee, N. Malouch, and S. Fdida, "Controlling spectrum handoff with a delay requirement in cognitive radio networks," in ICCCN, Aug. 2012.

[14] B. Kahraman and F. Buzluca, "An efficient and adaptive channel handover procedure for cognitive radio networks," Wireless Communication and Mobile Computing, Feb. 2013.

[15] C.-W. Wang and L.-C. Wang, "Modeling and analysis for proactive-decision spectrum handoff in cognitive radio networks," in Proc. IEEE ICC, 2009.

[16] Y. Song and J. Xie, "Common hopping based proactive spectrum handoff in cognitive radio ad hoc networks," in Proc. IEEE Globecom, Dec. 2010.

[17] " "Prospect: A proactive spectrum handoff framework for cognitive radio ad hoc networks without common control channel," IEEE Transactions on Mobile Computing, July 2012.

[18] C.-W. Wang, L.-C. Wang, and F. Adachi, "Modeling and analysis for reactive-decision spectrum handoff in cognitive radio networks," in Proc. IEEE Globecom, 2010.

[19] Y. Liu and M. Liu, "To stay or to switch: Multiuser multi-channel dynamic access," IEEE Transactions on Mobile Computing, Apr. 2015.

[20] F. Wang, M. Krunz, and S. Cui, "Priced-based spectrum management in cognitive radio networks," IEEE Journal of Selected Topics in Signal Processing, vol. 2, no. 1, pp. 74-87, 2008.

[21] M. Li, S. Salinas, P. Li, X. Huang, Y. Fang, and S. Glisic, "Optimal scheduling for multi-radio multi-channel multi-hop cognitive cellular networks," IEEE Transactions on Mobile Computing, Jan 2015.

[22] A. Lertsinsrubtavee, N. Malouch, S. Fdida, and K. Kanchanasut, "Spectrum sharing and impact on spectrum handoff in multichannel cognitive radio networks," in IEEE CORAL, June 2013.
[23] E. G. Coffman, Jr., D. S. Johnson, P. W. Shor, and R. R. Weber, "Markov chains, computer proofs, and average-case analysis of best fit bin packing," in Proceedings of the twenty-fifth annual ACM symposium on Theory of computing, ser. STOC '93, 1993.

[24] C. Kenyon, "Best-fit bin-packing with random order," in In 7th Annual ACM-SIAM Symposium on Discrete Algorithms, 1997.

[25] C. Cormio and K. R. Chowdhury, "A survey on MAC protocols for cognitive radio networks," Ad Hoc Networks, Sept. 2009.

[26] I. Marsic, Computer Networks Performance and Quality of Service. [Online]. Available: http://www.ece.rutgers.edu/ marsic/books/CN/, 2010.

[27] V. C. Ed., S. Das, L. Zhu, J. Malyar, and P. McCann, "Protocol to Access White-Space (PAWS) Databases," RFC 7545, May 2015.

[28] L. Yang, L. Cao, and H. Zheng, "Proactive channel access in dynamic spectrum networks," Physical Communication, vol. 1, no. 2, pp. 103-111, 2008

[29] T. C. Clancy and B. D. Walker, "Predictive dynamic spectrum access," in SDR Forum Technical Conference, 2006.

[30] "OMNeT++: A discrete event simulation environment for communication networks." [Online]. Available: http://www. omnetpp.org/

[31] "IBM ILOG CPLEX Optimization Studio." [Online]. Available: http://www-01.ibm.com/software/integration/ optimization/cplex-optimization-studio/

[32] R. K. Jain, D.-M. W. Chiu, and W. R. Hawe, "A quantitative measure of fairness and discrimination for resource allocation in shared computer system," Eastern Research Lab, Tech. Rep. DECTR-301, 1984.

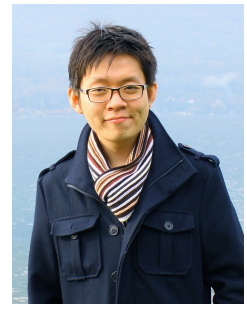

Adisorn Lertsinsrubtavee received the B.E. degree in telecommunication engineering from King Mongkut's Institute of Technology Ladkrabang, Bangkok, Thailand in 2006. He received the M.E. degree in Information and Communications Technologies from Asian Institute of Technology, Pathumthani, Thailand and the M.S. degree in Communication Networks and Services from Telecom Management SudParis, Evry, France in 2009. He received the Ph.D. degree in computer science from UPMC Sorbonne Universités, Paris, France in 2013. He is currently a research associate with the Computer Laboratory, University of Cambridge, UK. His current research interests includes cognitive radio networks, future internet architectures, and information centric networks.

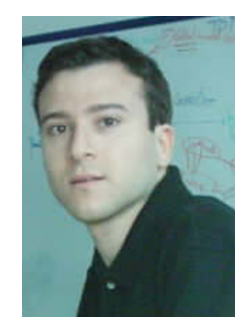

Naceur Malouch is an associate professor at the University Pierre et Marie Curie Paris 6 since 2003, and a researcher in the Network and Performance Analysis team at the computer science laboratory LIP6. He received a Ph.D. in computer science from the University of Nice SophiaAntipolis, France, in January 2003. During his Ph.D studies, he was with the MISTRAL group at INRIA Sophia-Antipolis. He was a cooperative student visitor at IBM T. J. Watson Research Center from June to August 2001. He obtained his engineering diploma in computer science from the Ecole Nationale des Sciences de Informatique (ENSI), University of Tunis II in June 1998. In the same year he was awarded the national prize of excellence in computer engineering. He was involved in several European and national projects dealing with wireless networks such as the European CELTIC BOSS project. He has been responsible for the research program of the Master degree from 2005 to 2007. His publications are in the area of transport protocols, overlay multicast, low-complexity highscalability QoS, and performance over wireless and mobile networks. He has served as TPC member in several international conferences such as GLOBECOM. He was a publicity co-chair of SIGMETRICS 2008. 